\title{
Optimization of dual-wavelength intravascular photoacoustic imaging of atherosclerotic plaques using Monte Carlo optical modeling
}

Nicholas Dana

Timothy Sowers

Andrei Karpiouk

Donald Vanderlaan

Stanislav Emelianov 


\title{
Optimization of dual-wavelength intravascular photoacoustic imaging of atherosclerotic plaques using Monte Carlo optical modeling
}

\author{
Nicholas Dana, ${ }^{a}$ Timothy Sowers,,${ }^{b, c}$ Andrei Karpiouk, ${ }^{d}$ Donald Vanderlaan, ${ }^{d}$ and Stanislav Emelianov ${ }^{\mathrm{d}, e, *}$ \\ aUniversity of Texas at Austin, Department of Biomedical Engineering, Austin, Texas, United States \\ ${ }^{\mathrm{b}}$ Georgia Institute of Technology, Parker H. Petit Institute for Bioengineering and Bioscience, Atlanta, Georgia, United States \\ 'Georgia Institute of Technology, George W. Woodruff School of Mechanical Engineering, Atlanta, Georgia, United States \\ ${ }^{\mathrm{d} G e o r g i a}$ Institute of Technology, School of Electrical and Computer Engineering, Atlanta, Georgia, United States \\ eEmory University School of Medicine, Georgia Institute of Technology, Wallace H. Coulter Department of Biomedical Engineering, Atlanta, \\ Georgia, United States
}

\begin{abstract}
Coronary heart disease (the presence of coronary atherosclerotic plaques) is a significant health problem in the industrialized world. A clinical method to accurately visualize and characterize atherosclerotic plaques is needed. Intravascular photoacoustic (IVPA) imaging is being developed to fill this role, but questions remain regarding optimal imaging wavelengths. We utilized a Monte Carlo optical model to simulate IVPA excitation in coronary tissues, identifying optimal wavelengths for plaque characterization. Near-infrared wavelengths $(\leq 1800 \mathrm{~nm})$ were simulated, and single- and dual-wavelength data were analyzed for accuracy of plaque characterization. Results indicate light penetration is best in the range of 1050 to $1370 \mathrm{~nm}$, where $5 \%$ residual fluence can be achieved at clinically relevant depths of $\geq 2 \mathrm{~mm}$ in arteries. Across the arterial wall, fluence may vary by over 10-fold, confounding plaque characterization. For single-wavelength results, plaque segmentation accuracy peaked at 1210 and $1720 \mathrm{~nm}$, though correlation was poor $(<0.13)$. Dual-wavelength analysis proved promising, with $1210 \mathrm{~nm}$ as the most successful primary wavelength $(\approx 1.0)$. Results suggest that, without flushing the luminal blood, a primary and secondary wavelength near 1210 and $1350 \mathrm{~nm}$, respectively, may offer the best implementation of dual-wavelength IVPA imaging. These findings could guide the development of a costeffective clinical system by highlighting optimal wavelengths and improving plaque characterization. @ 2017 Society of Photo-Optical Instrumentation Engineers (SPIE) [DOI: 10.1117/1.JBO.22.10.106012]
\end{abstract}

Keywords: intravascular; atherosclerosis; photoacoustic; clinical; Monte Carlo; spectroscopic.

Paper 170468R received Jul. 21, 2017; accepted for publication Sep. 28, 2017; published online Oct. 27, 2017.

\section{Introduction}

\subsection{Coronary Heart Disease and Treatment}

Coronary heart disease (CHD) affects $6.2 \%$ of American adults (aged $\geq 20$ years $)^{1}$ and occurs when lipid accumulates at the endothelium of the coronary arteries, resulting in the formation of an atherosclerotic plaque. ${ }^{2}$ This may result in luminal stenosis and/or develop into a vulnerable plaque, which can rupture, resulting in thrombus formation and myocardial infarction. ${ }^{2}$ Coronary-stent placement via percutaneous coronary intervention (PCI) is used to stabilize vulnerable plaques. ${ }^{3}$ Patient outcomes improve when PCI stenting-procedures utilize image guidance techniques, such as intravascular ultrasound (IVUS) imaging. ${ }^{4}$ Several imaging-related markers have been identified, such as fibrous cap thickness and necrotic core extent, ${ }^{5,6}$ which are key markers of rupture prone plaques. Unfortunately, no current clinical imaging system can reliably provide image guidance and assess these markers. This highlights the potential for image guidance to improve CHD patient outcomes, and photoacoustic (PA) imaging is one such modality being explored to fill this role.

\footnotetext{
*Address all correspondence to: Stanislav Emelianov, E-mail: stas@gatech
} .edu

\subsection{Intravascular Photoacoustic Imaging}

Intravascular photoacoustic (IVPA) imaging, combined with IVUS imaging, has been extensively leveraged as a means to provide image guidance and characterization of atherosclerotic plaques $^{7-16}$ and to evaluate coronary stent placement. ${ }^{17}$ This imaging modality utilizes the PA phenomenon to differentiate tissues based primarily on differences in their optical absorption. ${ }^{18,19}$ Results have been demonstrated using both endogenous ${ }^{7,9,13,14}$ and exogenous chromophores, ${ }^{10,20}$ prompting the development of IVPA imaging as a clinical imaging modality. ${ }^{11,16,21-23}$ Still, results have yet to decisively demonstrate which optical wavelength(s) provide optimal plaque characterization.

Ideally, to develop a clinical IVPA imaging system, one would conduct exhaustive experiments, preferably using IVPA imaging systems currently in development. ${ }^{11,16}$ This would require using multiple fresh coronary artery samples, with different disease states, with each imaged extensively with nearinfrared (NIR) excitation, followed by comprehensive histopathology to establish markers, such as luminal stenosis, plaque burden, and cap thickness. Unfortunately, such an exhaustive imaging study would prove difficult, though modeling methods offer a viable way to evaluate such a large parameter space.

1083-3668/2017/\$25.00 @ 2017 SPIE 


\subsection{Monte Carlo Modeling}

Monte Carlo (MC) optical modeling is a highly accurate modeling method, ${ }^{24}$ in which photon propagation in a medium is treated as a probabilistic process. It has been used to estimate fluence distributions in biomedical applications, ${ }^{25-29}$ including PA imaging of atherosclerotic plaques. ${ }^{30}$ We have adapted an MC model ${ }^{28}$ to simulate IVPA excitation across a range of NIR wavelengths, using several different tissue geometries. We leverage this model to provide insight into which IVPA system configurations may provide the best balance of contrast, imaging depth, and plaque characterization.

Spectroscopic imaging has already been established as a viable method for imaging lipid plaques in human tissues. ${ }^{13}$ However, spectroscopic imaging in a clinical setting would be prohibitively expensive due to the cost of laser source and prohibitively time-consuming due to the added acquisition time for the multiple wavelengths. Single-wavelength imaging presents the most economical and fastest option for lipid imaging yet it is likely to be the least accurate. We believe that dual-wavelength imaging would balance significant gains in the accuracy of lipid characterization with minimal increase to cost and acquisition time. Thus, we synthesized single- and dual-wavelength data sets, as these represent the most clinically translatable imaging systems. We intend the model-based results provided herein, which are supported by a simple experimental validation and corresponding simulation, to be used to inform IVPA imaging system development.

\section{Materials and Methods}

\subsection{Overview of Monte Carlo Model}

MCXLAB, the software used herein for optical modeling, was developed using CUDA ${ }^{31}$ by Fang and Boas ${ }^{28}$ and implemented in MATLAB ${ }^{\circledR}$ (MathWorks, Inc., Natick, Massachusetts). The simulation defines a three-dimensional (3-D) gridded model space, wherein each voxel designates the media comprising that specific location. Each medium is defined by four optical parameters: absorption $\left[\mu_{\mathrm{a}}\left(\mathrm{cm}^{-1}\right)\right]$, scattering $\left[\mu_{\mathrm{s}}\left(\mathrm{cm}^{-1}\right)\right]$, anisotropy $[g$ (unitless)], and refractive index [ $n$ (unitless)]. Nonreflective conditions were used at the model-exterior boundary. Collectively these define the optical properties of the entire simulation space. ${ }^{28}$ The model space used uniform grid spacing of $20 \mu \mathrm{m}$, which was approximately equivalent to the overall minimum mean free path (MFP) across the tissue types and wavelengths simulated. The result was a voxel volume of $20 \times 20 \times 20 \mu \mathrm{m}^{3}$, with each dimension spanning $8 \mathrm{~mm}$ total (400 voxels), for a total simulation volume of $8 \times 8 \times 8 \mathrm{~mm}^{3}$.

Illumination was modeled as a side-firing optical fiber located in the center of the model volume, approximating what has been described in previous IVPA experimental setups. ${ }^{11,13,16}$ The illumination pattern was modeled as a uniform cone (conicaxis parallel to the $X-Y$ plane), with a half-angle of $16.7 \mathrm{deg}$ $(0.292 \mathrm{rad})$. A side-firing ultrasonic transducer was modeled as being colocalized with the source, with the axis of ultrasonic propagation assumed to be colinear with optical conic-axis. Using previously reported parameters, ${ }^{16}$ the transducer sampling-volume, corresponding to the 3-D field of view of the transducer, was estimated in model space. The transducer beam profile was modeled for both the near-field and far-field zone according to the full-width half-maximum (FWHM) estimation. ${ }^{32}$ Figure 1(a) shows a 3-D overlay of the optical fiber, light cone, and transducer footprint, and a fluence cross section of a simulation done at $1000 \mathrm{~nm}$ in an aqueous medium is shown in Fig. 1(b).

\subsection{Consolidation of Model Output}

The output of a single simulation is a 3-D fluence distribution normalized to the simulated source energy, with each voxel containing one fluence value, $F(\vec{r}, \lambda)\left(\mathrm{mm}^{-2}\right)$. The PA signal at each voxel was modeled by taking the product of the fluence, the tissue optical absorption, and the Grüneisen parameter, $\Gamma(\vec{r}, T)$ (unitless) (measured at $22^{\circ} \mathrm{C}$ ), for water-based $(0.14)$ or lipid-based $(0.81)$ tissues $^{33}$ as appropriate. The result was a 3-D distribution of initial PA pressure amplitudes,
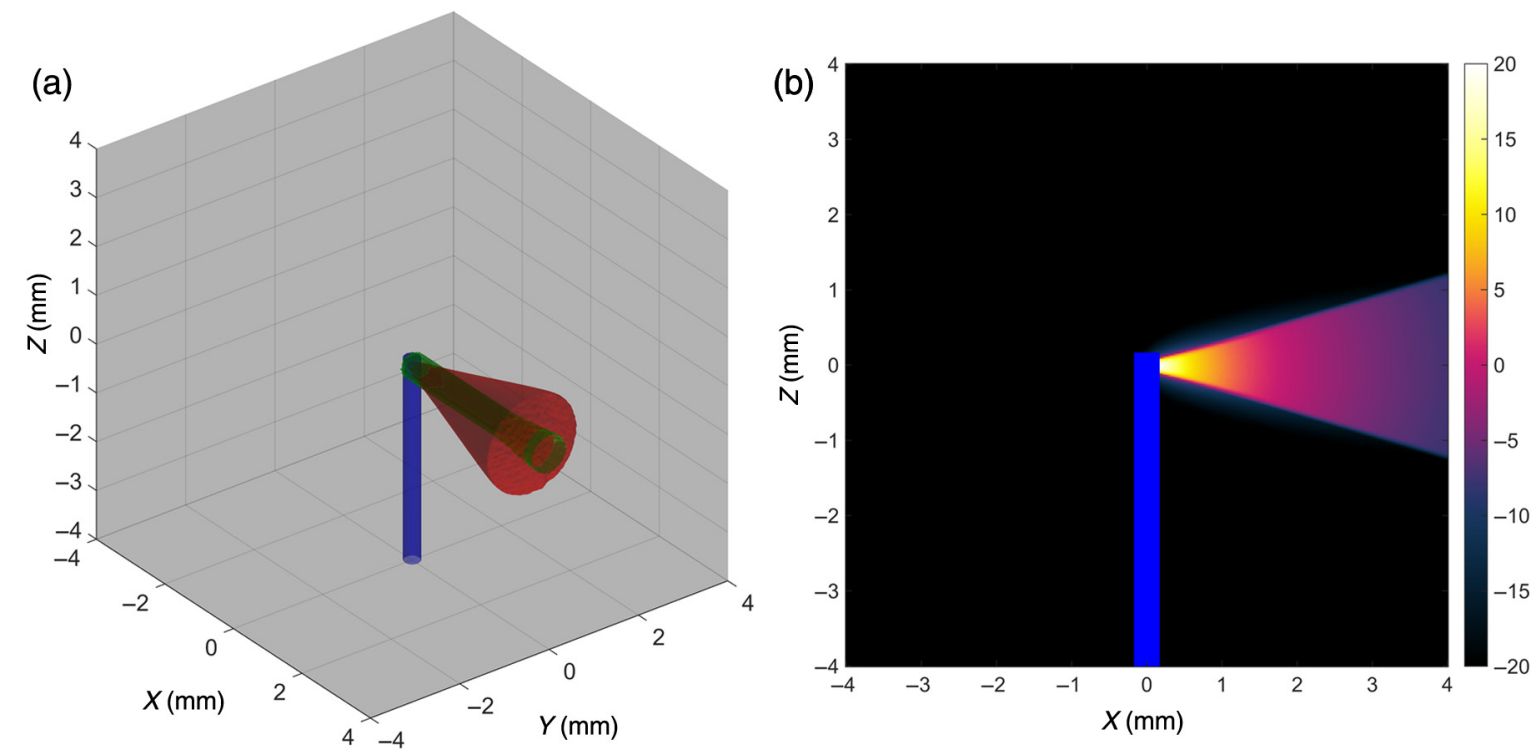

Fig. 1 (a) Volumetric rendering of optical fiber (blue), light cone (red), and transducer beam profile (green). (b) Fluence cross section ( $Y=0 \mathrm{~mm}, 40-\mathrm{dB}$ scale) of an optical simulation at $1000 \mathrm{~nm}$ in aqueous medium. 
$p_{0}(\vec{r}, \lambda, T)(\mathrm{Pa})$, equivalent to what is estimated by the PA equation. ${ }^{18,19}$ The initial PA pressure in voxels that coincided with the transducer beam profile was recorded. Ultrasonic propagation and attenuation were not directly simulated. The source and transducer were then rotated in the $X-Y$ plane, which is equivalent to rotating an IVPA imaging catheter in a coronary artery, and the simulation was repeated at the new angle. This was done every $2.81 \mathrm{deg}$ to sample the entire $360-$ deg arc in 128 steps. This scheme sampled the entire two-dimensional (2-D) $X-Y$ plane, with most voxels being sampled more than twice. Once the entire arc had been modeled and sampled, the PA signal from each of the 128 simulations was summed and normalized to the number of times each voxel was sampled. The result was an estimated 2-D distribution of PA signal sources $I_{\mathrm{PA}}(x, y, \lambda)$ as may be observed by IVPA imaging systems. Also recorded was the 2-D fluence distribution, $I_{F}(x, y, \lambda)$. This entire process was performed at wavelengths from 800 to $1800 \mathrm{~nm}$, in 10-nm steps, for each of four simulated tissue samples. Photon packets totaling $3 \times 10^{6}$ were launched for each A-line simulation, with the average runtime of a single A-line simulation being $12 \mathrm{~s}$ and the total runtime of all simulations totaling $172 \mathrm{~h}$. Simulations were performed on a Windows 7 (Microsoft, Seattle, Washington) computer with 16 GB of RAM, an Intel Core I7-4770 processor (Intel, Inc., Santa Clara, California), and a NVidia GTX 1080 GPU (NVidia, Inc., Santa Clara, California). The resulting data were comprised of an image at each of the 101 wavelengths for all four tissue types, resulting in a total of 404 simulated images.

\subsection{Tissue Models and Optical Properties}

Four 3-D tissue volumes were generated for the model. A recent study by Narula et al. ${ }^{6}$ examined 295 histopathologic samples of atherosclerotic coronary disease. Their results indicate that a cap thickness of $<54 \mu \mathrm{m}$ was the best indicator of plaque type, followed by macrophage infiltration and necrotic core area $\left(\geq 3.45 \mathrm{~mm}^{2}\right)$ and that percent luminal stenosis did not significantly differentiate stable from vulnerable plaques. ${ }^{6}$ Based on these results, we designed two vulnerable plaque models, one with a lipid pool that is diffusely distributed around the vessel (diffuse) and the second with a lipid pool confined to one side of the vessel (confined). Each consisted of a thin cap $(\leq 60 \mu \mathrm{m})$ and a large lipid pool $\left(\geq 3.9 \mathrm{~mm}^{2}\right)$, as would be expected in vulnerable plaques with varying degrees of stenosis. Conversely, a stable plaque (stable) was modeled containing a thicker cap $(\geq 80 \mu \mathrm{m})$ and a modest lipid pool $\left(\leq 2.4 \mathrm{~mm}^{2}\right)$. The final tissue model represents a healthy artery (streaks), with minimal fatty streaks and no stenosis. Other tissue properties, such as the thickness of intima, media, and adventitia, as well as overall diameter, were based on average values reported in literature. ${ }^{34}$ The region external to adventitia was modeled as pericardial (PC) fluid. ${ }^{35}$ The lumen of each vessel model was filled with whole blood at $95 \%$ oxygenation. Also, in each tissue sample, extending colinearly with the $Z$-axis for $4 \mathrm{~mm}$ was a $320-\mu$ m-diameter optical fiber, as would be needed for light delivery in a catheter implementation. Aside from the fiber optic extending partway into the tissue volume, the tissue samples did not vary along the $Z$-dimension. Figures 2 (a)-2(d) show a cross section of the four tissue types at the slice containing the tip of the optical fiber $(Z=0 \mathrm{~mm})$.

The optical properties of the seven tissue constituents in the NIR regime were tabulated from several sources, as described in
Table 1. Absorption data were extracted from the literature and, if needed, joined and smoothed to provide a continuous spectrum across the desired range. Scattering data were also extracted from the literature and smoothed by fitting data to a two-term function as suggested by Jacques. ${ }^{43}$ The optical properties of lipid were estimated based on measurements taken from adipose tissue, ${ }^{40-42}$ and fibrous cap properties were estimated based on measurements taken from the thin fibrous layer derived from human aorta. ${ }^{38} \mathrm{PC}$ fluid was modeled based on measurements taken from blood and plasma. ${ }^{39}$ All other tissue samples were modeled based on optical measurements taken from the tissues themselves, as shown in Table 1. Blood was assumed to have an oxygen saturation $\left(\mathrm{SpO}_{2}\right)$ of $95 \%$. At longer NIR wavelengths $(>1000 \mathrm{~nm})$, the optical absorption of most tissues closely mirrors that of water. ${ }^{43}$ Thus, due to the absence of explicit literature data at longer wavelengths, the optical absorption of intimal, medial, adventitial tissues, and PC fluid was estimated by linearly fitting available data to measurements made of sea water ${ }^{37}$ and extrapolating, as has been previously suggested. ${ }^{43}$ Figure 3 shows the final absorption and scattering values used in the model for the seven tissue types. The optical fiber was assumed to be minimally scattering and nonabsorptive at the modeled wavelengths. We estimated tissue anisotropy values based on data reported in literature but assumed that anisotropy for a given tissue varied by $<0.05$ across the wavelength range used, as was consistent for most sources. Since literature data indicated that the index of refraction for lipid and water is largely constant in the NIR, we treated the index of refraction for a given tissue as constant across the wavelengths modeled.

\subsection{Depth Penetration and Signal-to-Noise Analysis}

Once the outputs from the simulations were consolidated, several analysis methods were used. For the 2-D fluence distributions, optical penetration depth was assessed by sliding a $100 \times 100 \mu \mathrm{m}^{2}$ averaging kernel (to smooth abrupt changes in fluence due to tissue boundaries) along the dashed line in Fig. 2 and evaluating where fluence attenuated to $5 \%$ at each wavelength. The $5 \%$ fluence threshold corresponds to the probability of a photon transiting across three effective MFP lengths. ${ }^{44}$ In this case, the fluence would also be reduced from scattering due to the light being spread across a larger area as it propagates into the tissue. As such, it is a useful metric to compare between wavelengths and against other optical modeling results.

Signal-to-noise ratio (SNR) in the fluence estimates was calculated to quantify variation due to the number of simulated photons. This was done by sliding a $100 \times 100 \mu \mathrm{m}^{2}$ kernel across each $I_{F}(x, y, \lambda)$ image and calculating the mean fluence $\left[\overline{I_{F}}(x, y, \lambda)\right]$ and standard deviation $\left(\sigma_{F}\right)$ at each position. The SNR at that position was then taken to be the ratio of the two $\left[\overline{I_{F}}(x, y, \lambda) / \sigma_{F}\right]$, and then the mean SNR across the entire image was calculated for each wavelength and coronary model.

\subsection{Segmentation and Correlation Analysis}

To provide a useful metric to gauge the accuracy of the simulated results to visualize lipid, and thus plaque vulnerability, we implemented a simple segmentation algorithm on the simulated IVPA images followed by calculation of the Matthew's correlation coefficient $(\mathrm{MCC})^{45}$ to determine the quality of that imaging scheme to visualize plaque content. The segmentation 

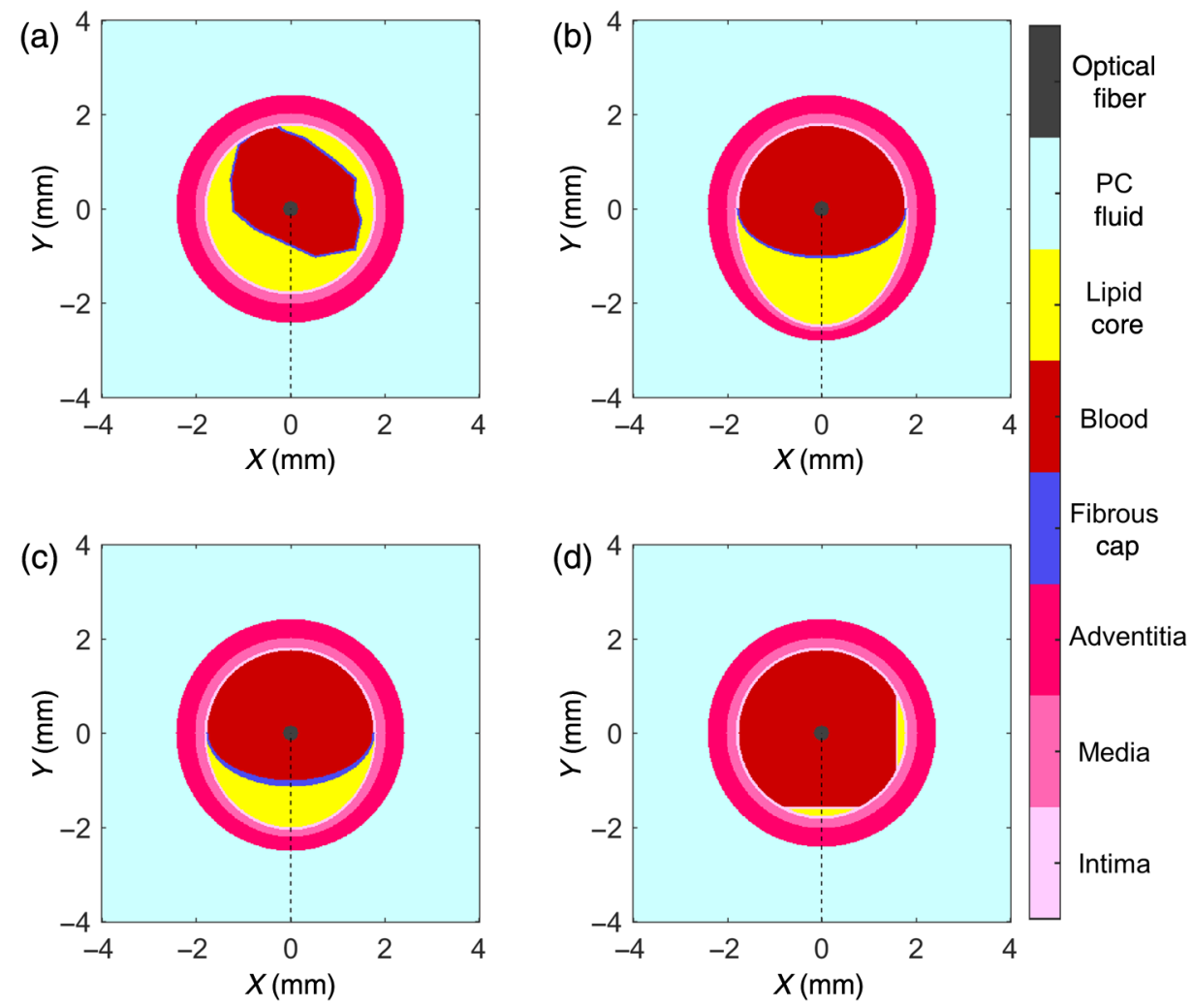

Fig. 2 Cross section of modeled tissue volumes at plane $Z=0 \mathrm{~mm}$. (a) Diffuse and (b) confined represent vulnerable plaques, with large lipid pools and thin caps. (c) Stable represents a stable plaque with a modest lipid pool and a thick cap. (d) Streaks represent a healthy artery with minimal lipid pool. Legend on the right denotes tissue types. Dashed line denotes location where light penetration analysis (Fig. 6) was completed.

algorithm used Otsu's method ${ }^{46}$ to apply a binary segmentation that designated each voxel of the fluence distribution as either lipid or nonlipid and then compared those results to the tissue cross-section image as ground truth. Voxels containing lipid were true (the yellow regions in Fig. 2) while all other voxels were false. We thus estimated true positive (TP), false positive (FP), true negative (TN), and false negative (FN) numbers for each image. These were used to determine the MCC based on the following equation:

$$
\mathrm{MCC}=\frac{\mathrm{TP} \times \mathrm{TN}-\mathrm{FP} \times \mathrm{FN}}{\sqrt{(\mathrm{TP}+\mathrm{FP})(\mathrm{TP}+\mathrm{FN})(\mathrm{TN}+\mathrm{FP})(\mathrm{TN}+\mathrm{FN})}} .
$$

An MCC of 1 indicates a perfect match between true and measured positive and negative values, while an MCC of -1 indicates a perfect reversal of positive and negative matches, with zero representing no correlation. A value for the MCC was calculated for each of the 101 wavelengths, $\operatorname{MCC}(\lambda)$, and for each coronary model. A composite MCC was also calculated at each wavelength by aggregating the TP, TN, FP, and FN values from all four coronary models.

\subsection{Ratiometric-Image Analysis}

Additionally, given that a dual-wavelength IVPA imaging system is the most straight forward implementation of a multiwavelength IVPA imaging platform, analysis was done to determine which wavelength pairs may provide the best assessment of plaque content. A simple wavelength ratiometric method was applied to generate a ratio image, $I_{\mathrm{PA}}^{R}\left(x, y, \lambda_{1}, \lambda_{2}\right)=$ $I_{\mathrm{PA}}\left(x, y, \lambda_{1}\right) / I_{\mathrm{PA}}\left(x, y, \lambda_{2}\right)$. This image was then linearly scaled to within $[0,1]$ and processed using the same segmentation method described above. A single MCC was calculated for each $I_{\mathrm{PA}}^{R}\left(x, y, \lambda_{1}, \lambda_{2}\right)$ image. The result is a $101 \times 101$ (wavelength $\times$ wavelength) MCC heatmap for each coronary model and a final composite MCC heatmap using the TP, TN, FP, and FN data across all $I_{\mathrm{PA}}^{R}\left(x, y, \lambda_{1}, \lambda_{2}\right)$ image segmentations.

\subsection{Experimental Validation}

A simulation and experiment were completed to validate the performance of the modeling method. Porcine lipid was placed at the top surface of a phantom consisting of $4 \%$ gelatin from porcine skin (Sigma, G2500-1KG) and water. The PA signal was collected with an IVUS/IVPA catheter (diameter $500 \mu \mathrm{m}$, center frequency $40 \mathrm{MHz}$, and bandwidth $50 \%)^{16}$ at 28 wavelengths of interest between 1200 and $1750 \mathrm{~nm}$, using an average of $2 \mathrm{~mJ} /$ pulse. This wavelength range was chosen because it is of most interest based on the results of the simulations in the four coronary arteries. The A-line was sampled at $200 \mathrm{MHz}$ and filtered using a bandpass filter between 10 and $60 \mathrm{MHz}$. Then, 20 of these A-lines were averaged, and a 10-dimensional $(0.075 \mathrm{~mm})$ median filter was applied spatially across the A-line to reduce noise. Next, a ratiometric A-line for each possible wavelength pair was calculated. The ratiometric A-lines were 
Table 1 Comparison of tissue optical parameters for absorption, $\mu_{\mathrm{a}}(\lambda)\left(\mathrm{cm}^{-1}\right)$, scattering, $\mu_{\mathrm{s}}(\lambda)\left(\mathrm{cm}^{-1}\right)$, anisotropy, $g(\lambda)$ (unitless), and index of refraction, $n$ (unitless), as well as the data sources used to estimate values. Blood was assumed to be $95 \%$ oxygen saturated. Anisotropy values typically varied by $<0.05$ across the wavelength range used for a given tissue type. Index of refraction was assumed to be constant with respect to wavelength for each tissue type.

Tissue optical parameters and sources (values estimated at $1210 \mathrm{~nm}$ )

\begin{tabular}{|c|c|c|c|c|c|}
\hline Tissue type & $\mu_{\mathrm{a}}(\lambda)\left(\mathrm{cm}^{-1}\right)$ & $\mu_{\mathrm{s}}(\lambda)\left(\mathrm{cm}^{-1}\right)$ & $g(\lambda)$ & $n$ & Sources \\
\hline \multirow[t]{2}{*}{ Intima } & 0.120 & 9.98 & 0.89 & 1.40 & 36 \\
\hline & & & & & 37 \\
\hline \multirow[t]{2}{*}{ Media } & 0.155 & 16.1 & 0.86 & 1.40 & 36 \\
\hline & & & & & 37 \\
\hline \multirow[t]{2}{*}{ Adventitia } & 0.256 & 13.1 & 0.89 & 1.40 & 36 \\
\hline & & & & & 37 \\
\hline Fibrous cap & 0.453 & 15.8 & 0.90 & 1.40 & 38 \\
\hline \multirow[t]{2}{*}{ Blood } & 0.132 & 42.1 & 0.94 & 1.35 & 37 \\
\hline & & & & & 39 \\
\hline \multirow[t]{3}{*}{ Lipid } & 0.181 & 15.2 & 0.93 & 1.47 & 40 \\
\hline & & & & & 41 \\
\hline & & & & & 42 \\
\hline \multirow[t]{2}{*}{ PC fluid } & 0.100 & 0.0421 & 0.80 & 1.34 & 37 \\
\hline & & & & & 39 \\
\hline
\end{tabular}

then binarized using Otsu's method. Finally, since the porcine lipid behaves as a homogenous absorber, the MCC was calculated for the region $0.3 \mathrm{~mm}$ from either side of the water/lipid interface. This length consisted of the region immediately before and during the expansion phase of the PA response in the porcine lipid. $^{47}$

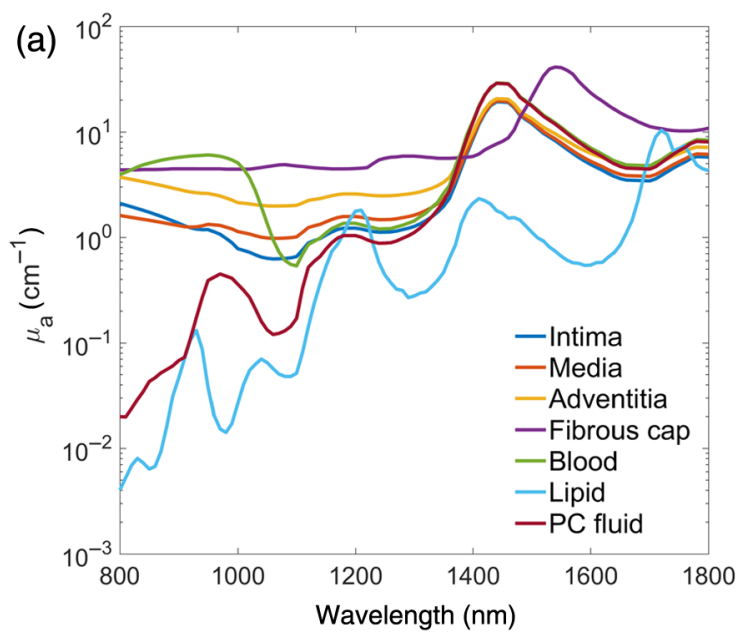

This experiment was then simulated using MCXLAB. The experiment was modeled using a $12 \times 12 \times 12 \mathrm{~mm}^{3}$ volume generated with a voxel size of $30 \mu \mathrm{m}$. The model space was uniformly water up to a depth of $1.25 \mathrm{~mm}$ and lipid at greater depths, which matched the experimental phantom used. Fluence distributions were found using simulations at the same wavelengths used in the experiment. PA lines were generated by multiplying the fluence distribution from the model with the Grüneisen and absorbance coefficient for the material at each voxel. As in the experimental analysis, a ratiometric A-line for each wavelength pair was calculated and then binarized into lipid and nonlipid components using Otsu's method. Finally, the MCC was calculated for each wavelength pair using the voxels in the region $0.3 \mathrm{~mm}$ from either side of the water/lipid interface.

\section{Results}

\subsection{Results of Experimental Validation}

The MCC plots for the experimental validation and corresponding simulation are shown in Figs. 4(a) and 4(b), respectively. The experiment resulted in high MCC values when wavelengths near the 1210- and 1720-nm absorption peaks of lipid were used. The shorter imaging depth and less diverse number of chromophores meant that the fluence distribution in the experiment and simulation had less variation than in the modeling of the four coronary arteries. Because of this and lack of noise, the model showed nearly all wavelength combinations giving either perfectly positive $(+1)$ or negative $(-1)$ MCC values. The optimal wavelength pairs in the experimental results agree with the corresponding simulation. Seventeen wavelength pairs had MCC values above 0.9 for the experiment. Of these 17 wavelength pairs, 15 had a primary wavelength near 1720 and 14 had a secondary wavelength in the range of 1540 to $1640 \mathrm{~nm}$. All 17 of these pairs had MCC values of 1 in the model, indicating perfect correlation.

\subsection{Signal-to-Noise Results}

SNRs were evaluated to indicate that a sufficient number of photons were used in the MCX simulations. The mean SNR in the fluence distributions, $I_{F}(x, y, \lambda)$, across all wavelengths and

Fig. 3 Optical (a) absorption and (b) scattering coefficients used to model tissue types. Sources for data can be found in Table 1.

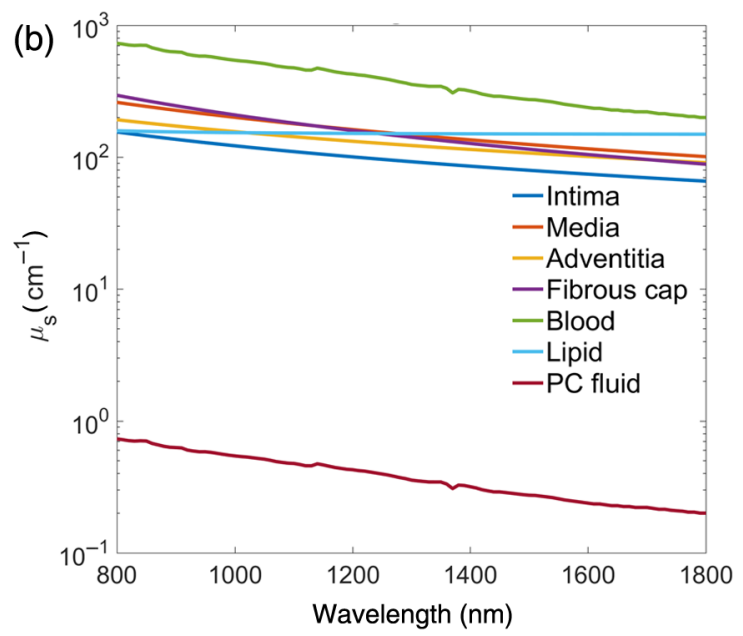



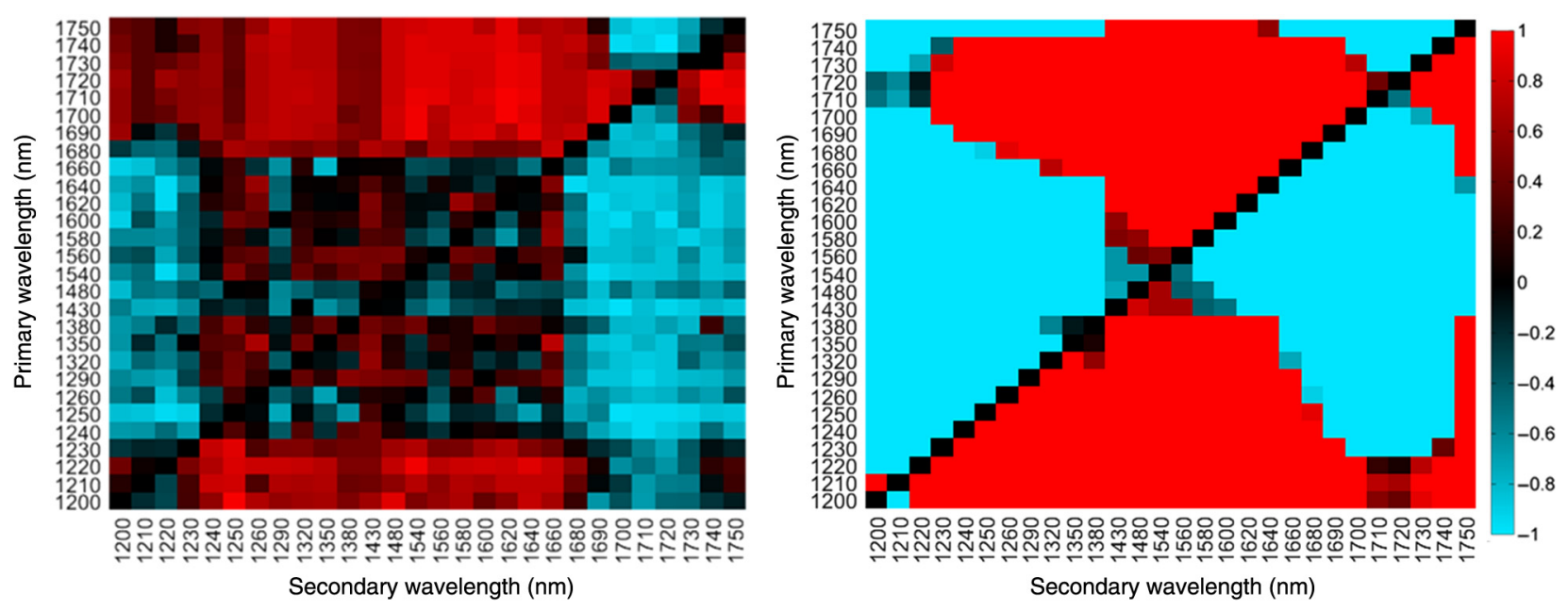

Fig. $4 \mathrm{MCC}$ color maps from the validation experiment indicating the accuracy of each two-wavelength pair for correctly identifying lipid. (a) Results from the experiment with porcine lipid. (b) Results of the corresponding simulations. Both show accurate lipid identification occurring with primary wavelengths near the 1210- and 1720-nm absorption peaks of lipid. Positive correlations are shown in red while negative correlations are shown in blue.

tissue geometries was $12.6 \pm 2.2$ and never fell below 6.3 for any simulated wavelength or coronary model.

\subsection{Visualization of Simulated IVPA Results}

Figure 5 shows simulated IVPA data from the four coronary models at $1210 \mathrm{~nm}$, displayed in $\mathrm{dB}$ scale. Optical absorption of lipid at $1210 \mathrm{~nm}$ is a local maximum and exceeds that of water [as seen in Fig. 3(a)], which contributes to the high PA signal seen in the lipid regions in Fig. 5 (lipid regions are shown in yellow in Fig. 2). Despite the high contrast between lipid and water-based tissues seen in Fig. 5, the optical fluence varies by more than an order of magnitude across the lumen and arterial wall.

\subsection{Depth Penetration and Single-Wavelength Segmentation Analysis}

Figure 6 shows the results of the depth penetration analysis (a) and the single-image segmentation results (b). The $5 \%$ fluence threshold varied between 1.5 and $2 \mathrm{~mm}$ for most wavelengths examined, but the wavelength window from $\sim 1050$ to $1350 \mathrm{~nm}$ showed best overall depth penetration. The MCC values shown in Fig. 6(b) indicate modest correlation, with the wavelength regions near 1210 and $1720 \mathrm{~nm}$, which correspond to relative peaks in the lipid optical absorption, resulting in the best correlation. This agrees with previous studies, which have found IVPA imaging contrast between lipid and water is best at these wavelengths. ${ }^{7,9,13,14,48}$

\subsection{Results of Ratiometric Analysis and Segmentation}

Figure 7 shows an example of the ratiometric image segmentation process and results. $I_{\mathrm{PA}}(x, y, \lambda)$ images are shown on the same linear scale at 1210 [Fig. 7(a)] and $1350 \mathrm{~nm}$ [Fig. 7(b)]. Note that at $1350 \mathrm{~nm}$, the optical absorption of water is roughly an order of magnitude greater than that of lipid [Fig. 3(a)], resulting in strong light absorption near the source at this wavelength as compared to $1210 \mathrm{~nm}$. For this reason, the corresponding ratiometric image [Fig. 7(c)] highlights the lipid-filled regions. As a result, the segmented image, as shown in Fig. 7(d), strongly correlates to the lipid-filled regions in Fig. 2(a).

\subsection{Correlation Heatmap Results}

Results from the image segmentation and correlation analysis on the ratiometric images are shown in Fig. 8 for all four coronary models, as well as a composite MCC heatmap [Fig. 8(e)]. A variety of dual wavelength combinations resulted in very high (>0.95) correlation. In general, results suggest that using a primary wavelength near 1210 or $1720 \mathrm{~nm}$ showed good correlation using a range of secondary wavelengths, as evidenced by the horizontal bands (red) centered near those wavelengths and present in all heatmaps shown in Fig. 8.

\section{Discussion}

\subsection{Limitations of Current Study}

It should be noted that the results demonstrated by this work represent an idealized model rather than the expected results from a realistic imaging setup. This model reconstructed the optical absorber map to a very high resolution $(20 \mu \mathrm{m})$ that is unavailable to common IVUS systems. While the findings do not rely on accurate segmentation of very small anatomical features $(<80 \mu \mathrm{m})$, the spatial reconstructions shown herein are unavailable to common IVPA systems, which typically have a spatial resolution near $60 \mu \mathrm{m} .{ }^{16}$ Furthermore, the model did not account for the frequency-dependent attenuation of PA signals as they transit through tissue, the introduction of image artifacts due to variations in the speed of sound or ultrasonic refraction at media boundaries, signal loss in the transducer Fresnel zone, the transfer function of the detector system, or electrical noise within measured signals. ${ }^{32}$ These factors will degrade image quality to a varying extent, and thus the results presented here are a generalized ideal. Future work could adapt and couple these results to an appropriate acoustic model that accounts for 

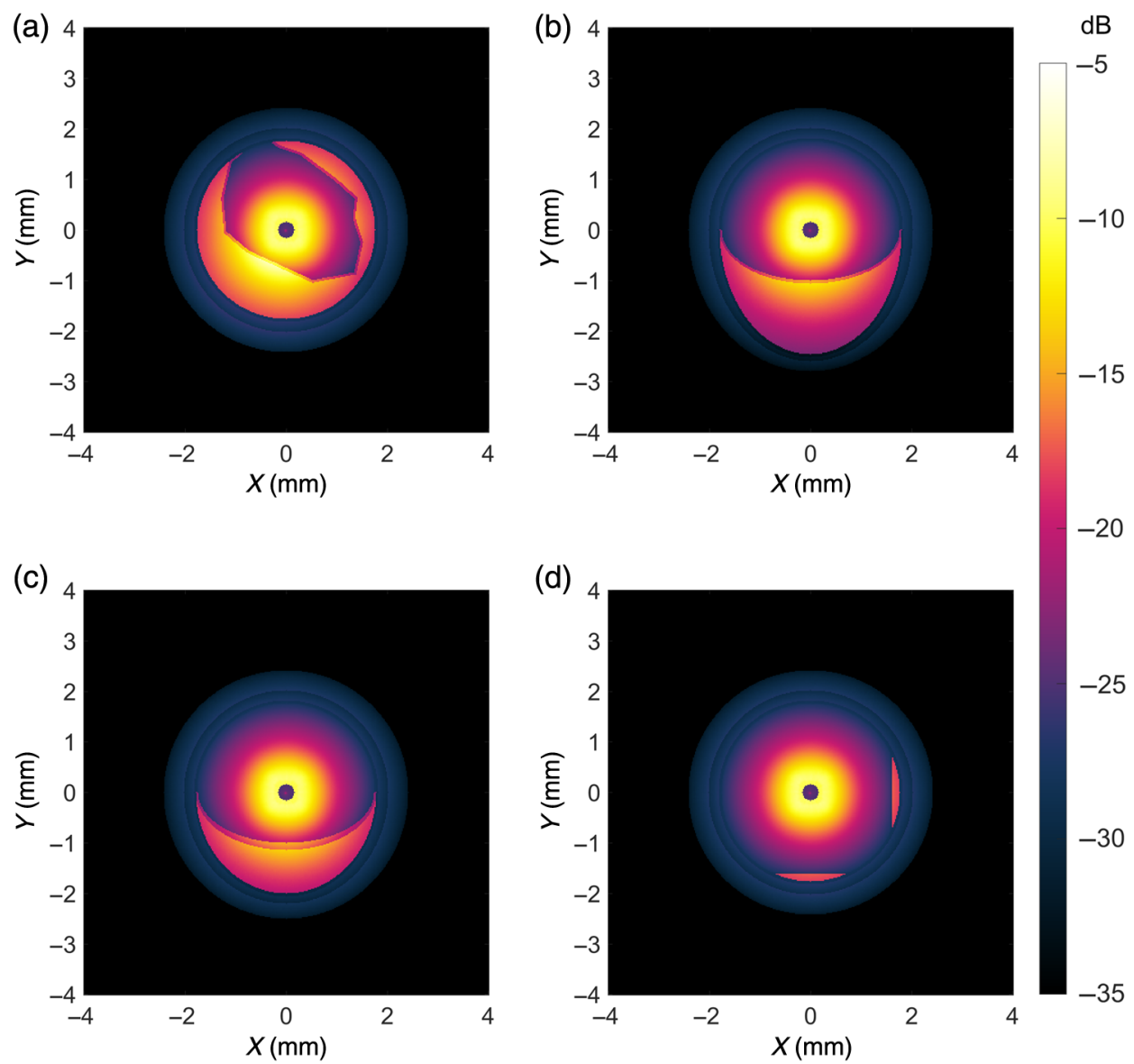

Fig. 5 Simulated PA data at $1210 \mathrm{~nm}$, for (a) diffuse, (b) confined, (c) stable, and (d) streaks coronary models. Images are presented on $\mathrm{dB}$ scale. Note that $1210 \mathrm{~nm}$ represents a local absorption-maxima of lipid over water which is demonstrated by the high-intensity signals in the lipid regions.
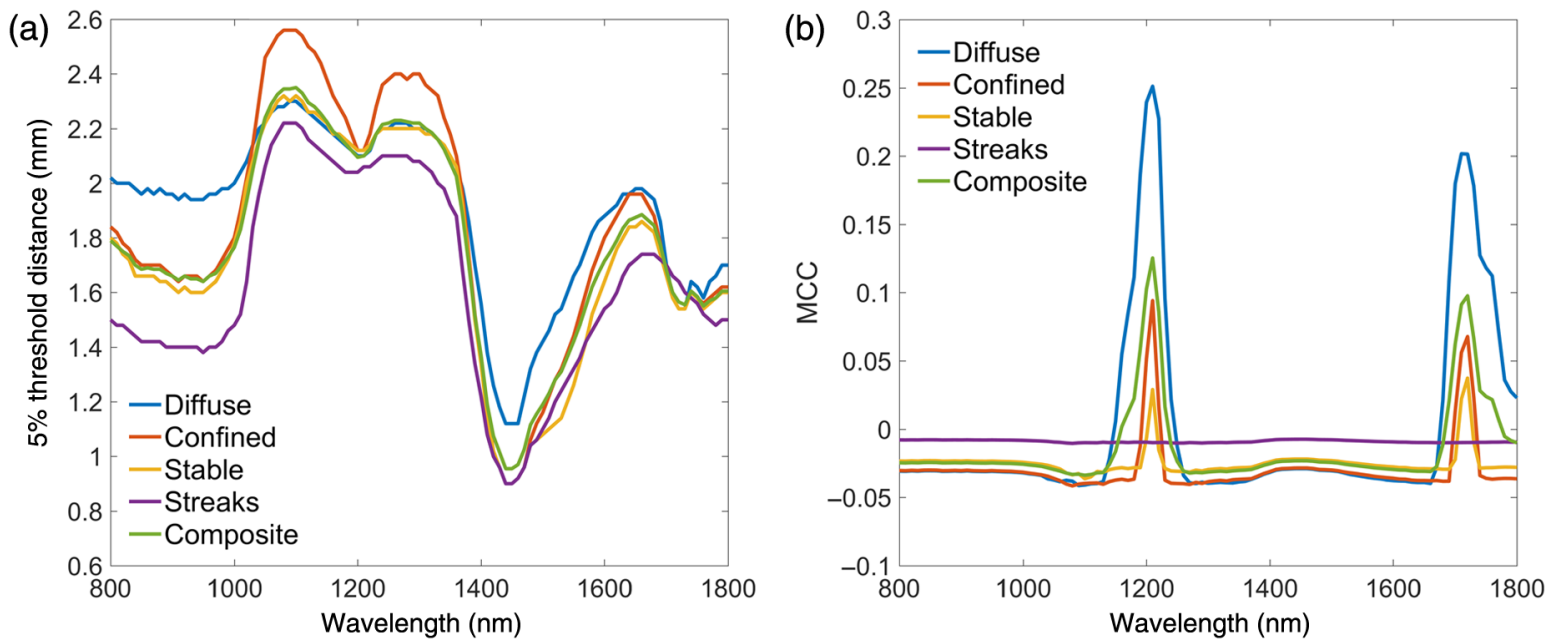

Fig. 6 (a) Analysis of light penetration across the four tissue types and the composite results. A 5\% normalized fluence threshold was determined for each coronary model and wavelength. Analysis was done along the dashed line in Fig. 2, which corresponds to the approximate center of the lipid-rich region. (b) Correlation analysis for the segmented single-wavelength data for the four coronary models and composite results. Two wavelength regions stand out, near 1210 and $1720 \mathrm{~nm}$, which correspond to the local absorption maxima of lipid over water. 

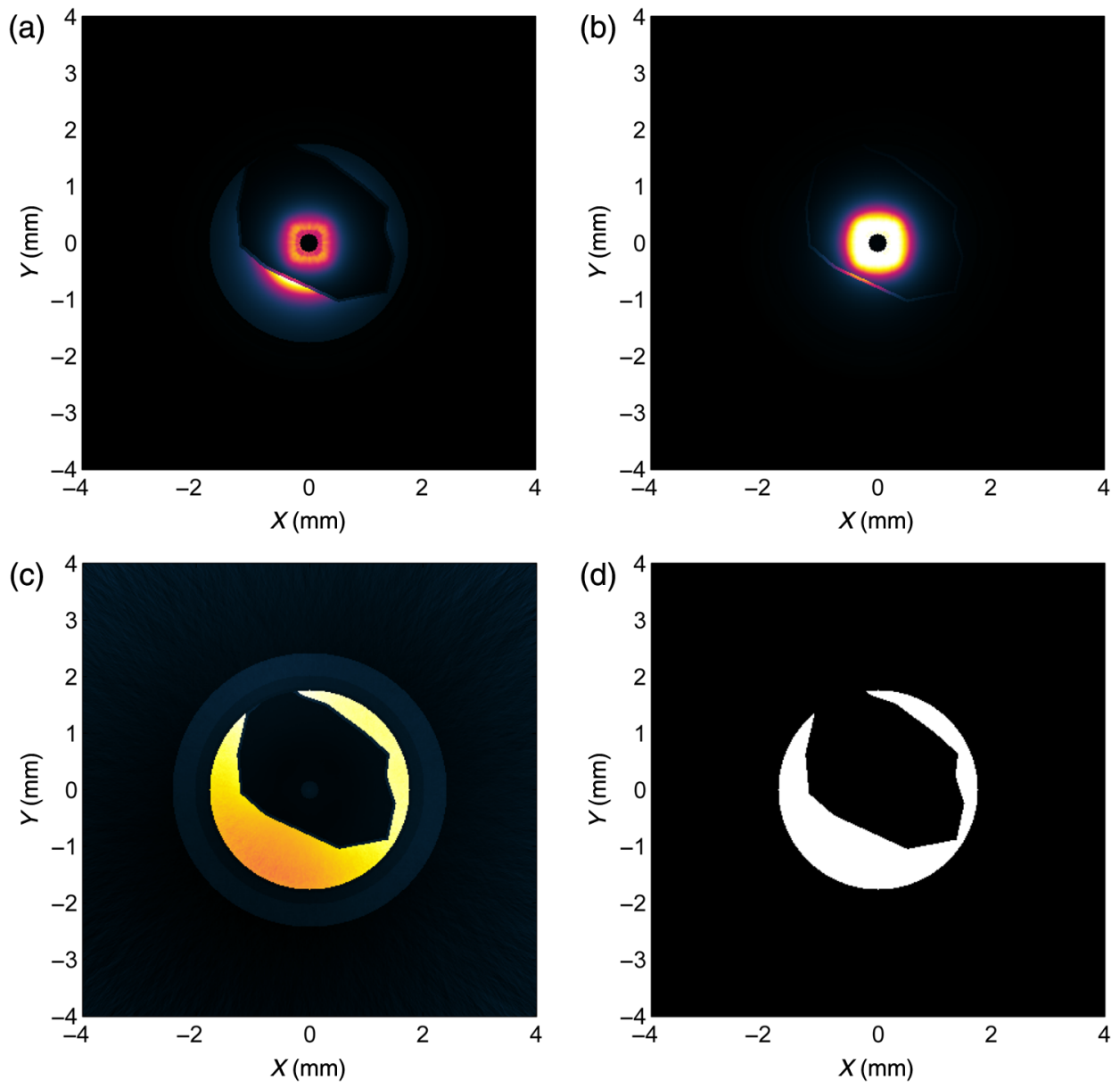

Fig. 7 Simulated PA data, $I_{\mathrm{PA}}(\lambda)$, for diffuse coronary model at (a) $1210 \mathrm{~nm}$ and (b) $1350 \mathrm{~nm}$. Images are displayed on the same linear scale for comparison. Ratiometric image, $I_{\mathrm{PA}}^{R}\left(\lambda_{1}, \lambda_{2}\right)$, using data displayed in (a) and (b), shown on a linear scale in (c). Segmented image based on (c), with predicted lipid-rich regions shown as white in (d).

these various factors, which would represent a significant iteration on these results.

\subsection{Experimental Validation Discussion}

High MCC values were attained for the experimental validation [Fig. 4(a)] when wavelengths near the 1210- and 1720-nm absorption peaks of lipid were used. The corresponding modeling indicated perfectly positive $(+1)$ or perfectly negative $(-1)$ MCC values for almost every wavelength combination. This is due to a lack of noise, and because the shorter imaging depth and less diverse number of chromophores meant that the fluence distribution in the experiment and corresponding simulation had less variation than in the modeling of the four coronary arteries. Despite these differences, the best wavelength pairs in the experiment agree with those from the corresponding model, with the 17 best wavelength pairs (MCC $>0.9$ ) from the experiment all having perfect MCC values of 1 in the corresponding model. These results support the fact that the general approach used for this study - determining a PA pressure distribution via $\mathrm{MC}$ modeling and then segmenting a ratiometric image to locate lipid-is a valid one.

Out of the 17 best wavelength pairs, 15 had a primary wavelength near 1720 and 14 had a secondary wavelength in the range of 1540 to $1640 \mathrm{~nm}$. We hypothesize that the higher absorption coefficient near the 1720-nm lipid peak resulted in the strongest signal for the validation experiment. Further, the secondary wavelength ranges from 1540 to $1640 \mathrm{~nm}$ gave the best results due to the large difference in water and lipid absorption coefficients. However, it should be noted here that the high absorption coefficient in both these wavelength bands can make them less effective when interrogating samples at greater depth due to a reduction in fluence. This proved to be the case when modeling the four coronary arteries.

\subsubsection{Signal-to-noise ratio and computation time}

The fact that the mean SNR never fell below 6.3 across all simulations suggests that the number of photon packets launched and the computation time were sufficient to provide modeled fluence distributions with minimal discontinuities and noise. The smoothness of that data can be seen in Fig. 5, where sharp variations in intensity are visible only intermittently at tissue boundaries.

\subsection{Discussion of General Imaging Results}

A primary finding of this study is that wavelength-dependent variation in fluence distribution across the tissue and between wavelengths can greatly affect the observed PA ratio, and thus the characterization of plaques. When applying a ratiometric 

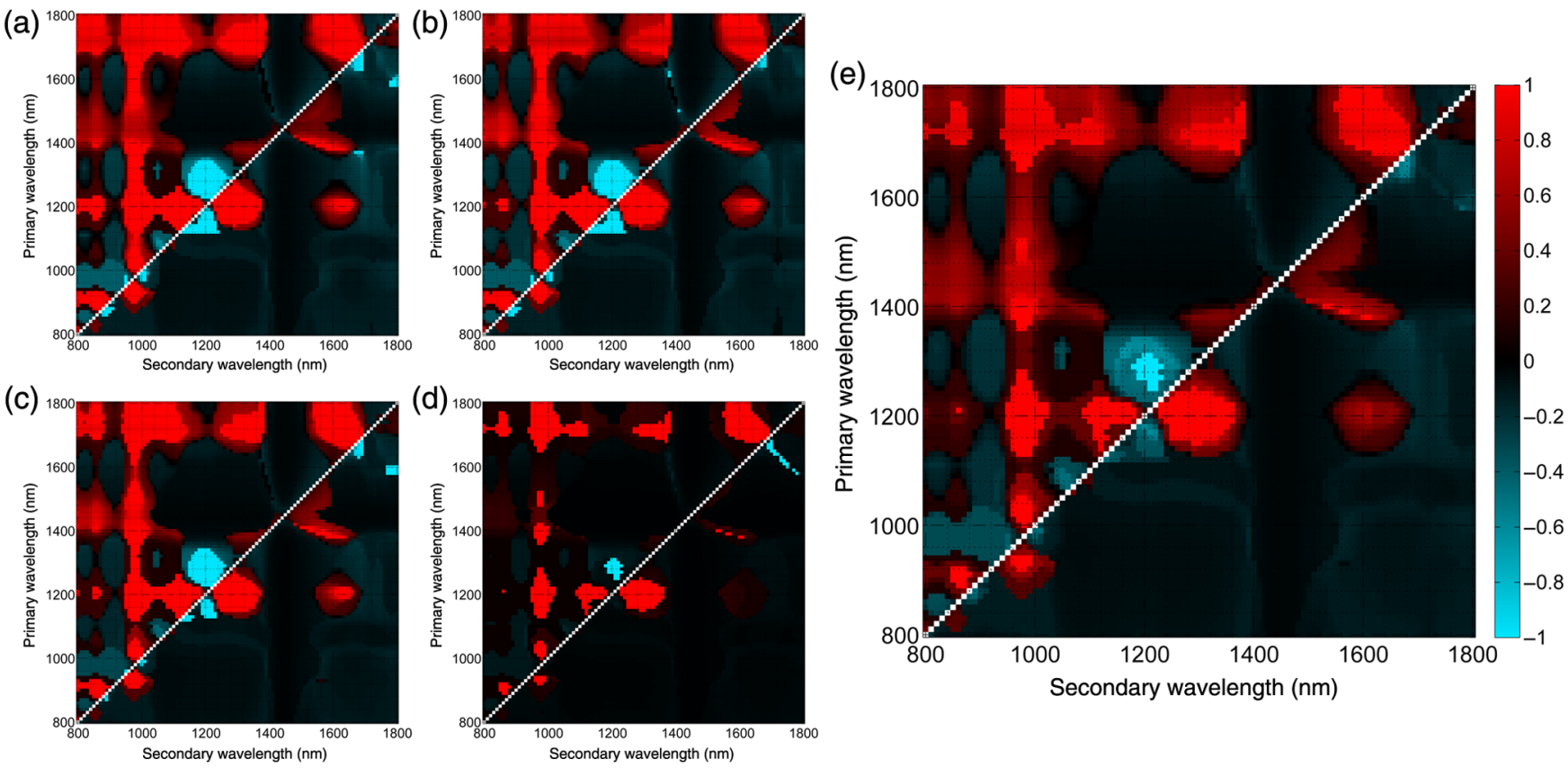

Fig. $8 \mathrm{MCC}$ heatmap, $\operatorname{MCC}_{\mathrm{hm}}\left(\lambda_{1}, \lambda_{2}\right)$, data from analysis of ratiometric images, $I_{\mathrm{PA}}^{-}\left(x, y, \lambda_{1}, \lambda_{2}\right)$, for (a) diffuse, (b) confined, (c) stable, and (d) streaks tissue coronary models, as well as composite MCC analysis in which the TP, TN, FP, and FN of all four models at each wavelength are aggregated to calculate MCC (e). The left and bottom axes represent the primary $\left(\lambda_{1}\right)$ and secondary $\left(\lambda_{2}\right)$ wavelengths used, respectively. All images are on the same scale, shown at the right of (e).

analysis, contrast for a given ROI is determined by the ratio of PA pressure generated using two different imaging wavelengths. When fluence is constant across wavelengths, the ratio of PA pressure is determined solely by the wavelength-dependent absorption coefficient. However, the assumption of constant fluence across wavelengths is highly circumspect. For example, 1210 and $1300 \mathrm{~nm}$ show comparable penetration depths [Fig. 6(a)], suggesting fluence variation between these wavelengths is small. This implies ratiometric contrast is largely determined by the absorption coefficient. This is not the case when pairing $1210 \mathrm{~nm}$ with $1420 \mathrm{~nm}$, whose penetration depths vary by a factor of 2, due largely to the increased optical absorption of water at $1420 \mathrm{~nm}$ [Fig. 6(a)]. Consequently, when using this wavelength combination, the PA pressure-ratio will be in some regions increased, and in others decreased, due to the fluence variation between wavelengths. As such, this wavelength combination will generally serve as poor indicator of the molecular composition within the tissue. Note that these effects become more pronounced at greater imaging depths, where the accumulation of optical losses is increased.

While not completely unexpected, this finding runs contrary to an ideal imaging modality, one whose contrast depends solely upon the imaging target (i.e., its tissue optical absorption) but not on other factors, such as the wavelength-dependent differences in fluence distribution seen here. For accurate segmentation to occur, contrast between plaque lipid and other tissues must remain robust under the corrupting influence of noise, image artifacts, and depth-dependent fluence attenuation, which cannot occur in regions where fluence has been reduced to near zero. This is an even more pressing concern in clinical practice, as the placement of the IVPA catheter in the artery center cannot be assured, which may mean the necessity to image through an even larger blood volume than modeled herein. The depthdependent fluence attenuation could also be affected by natural physiological variability in the size of different patients. Finally, depth-dependent fluence variations would also be affected if the artery is flushed with saline, which would be similar to common practice in OCT imaging. IVUS imaging may partly allay these concerns, as it can provide additional context to morphology and tissue content. ${ }^{8}$ It may even be possible to leverage morphology obtained from IVUS imaging to partially account for anticipated fluence variations, though this would require making some assumptions about imaged tissues optical properties.

\subsection{Optical Depth Penetration and Wavelength Selection}

As can be seen in Fig. 6(a), the 5\% fluence threshold exceeded $2 \mathrm{~mm}$, on average, in the wavelength region from 1050 to $1370 \mathrm{~nm}$. Given that the average coronary artery has a diameter of $\sim 3.5 \mathrm{~mm},{ }^{34}$ this wavelength window may represent the optimal imaging region in terms of achieving sufficient light delivery throughout the coronary artery. At wavelengths below $1050 \mathrm{~nm}$, the increased optical absorption of hemoglobin $(\mathrm{Hb})$ in blood [Fig. 1(a)] strongly attenuates light, as seen in the analysis of the streaks coronary model (purple line), which shows reduced depth penetration across most wavelengths. Additionally, the high optical absorption of water at wavelengths past $1370 \mathrm{~nm}$ results in similar light attenuation, this time across all coronary models. It is serendipitous that the 1210-nm optical absorption peak of lipid should fall in the middle of the window of reduced optical attenuation. Though the optical absorption difference (contrast) between lipid and water is greater at $1720 \mathrm{~nm}$ than at $1210 \mathrm{~nm}$ [Fig. 1(a)], given the overall strong light attenuation at the former wavelength, achieving real-time IVPA imaging with sufficient SNR across the entire imaging area at $1720 \mathrm{~nm}$ may prove challenging. In addition, the higher pulse energies required at $1720 \mathrm{~nm}$ 
could lead to heating of the tissue, an issue that has still not been comprehensively addressed by the IVPA imaging field. This analysis suggests that efforts should focus on developing IVPA imaging systems designed to operate in the 1050- to 1370$\mathrm{nm}$ range. Still, as laser pulse energy and ultrasonic-detector sensitivity vary across systems, it may be possible to achieve adequate IVPA signal generation at increased depths while imaging at $1720 \mathrm{~nm}$.

\subsection{Single-Wavelength Correlation Discussion}

Overall MCC values for single-wavelength images showed peaks at $0.125(1210 \mathrm{~nm})$ and at $0.098(1720 \mathrm{~nm})$. While these findings confirm previous results that indicated 1210 and $1720 \mathrm{~nm}$ optimized lipid-water contrast, ideal correlation values would be near 1 . The most likely reason for the low MCC values is that, at most wavelengths, the fluence spatial variations exceed those of optical absorption, and thus dominate the imaging results. While easy to account for visually, this is likely to confound an automated segmentation algorithm like the one used, which attempts to separate the entire image-space into a bimodal histogram for segmentation. ${ }^{46}$ Indeed, in addition to IVPA imaging at multiple wavelengths, it may be possible to implement an adaptive algorithm that significantly improves segmentation accuracy. Once a clinical IVPA imaging system is in further development and optimal imaging wavelength(s) are selected, a more sophisticated segmentation algorithm could be tested and implemented.

\subsection{Ratiometric Image and Heatmap Discussion}

Figures 7(a) and 7(b) show simulated imaging results obtained at different wavelengths, with contrast between the images due to differences in both the fluence distribution, as well as optical absorption. This highlights the fact that selection of an IVPA imaging wavelength must strike a balance between optical absorption, depth penetration, and fluence variation. Since previous studies have indicated the potential of IVPA imaging at 1210 and $1720 \mathrm{~nm},{ }^{7,9,13,14,48}$ it is not surprising that these primary wavelengths $\left(\lambda_{1}\right)$ show the most promise across all correlation heatmaps. The peak composite MCC occurs at $\lambda_{1}=1210 \mathrm{~nm}$ and $\lambda_{2} \approx 1350 \mathrm{~nm}$, with several MCC values at unity in this wavelength region. The optical absorption of water at $1350 \mathrm{~nm}$ exceeds that of fat by a factor of 10 . Thus, by taking the ratio of the signal at a strong lipid absorbing wavelength and the signal at a wavelength where water exhibits strong absorption, the PA signal from lipid regions is emphasized. This is further evidenced by the region of high correlation at $\lambda_{1}=1720 \mathrm{~nm}$ and $\lambda_{2} \approx 1600 \mathrm{~nm}$, where at $1600 \mathrm{~nm}$ the optical absorption of water again exceeds that of lipid by a factor of 10 . While this rationale suggests that a secondary wavelength be selected that best highlights water over lipid [e.g., $1460 \mathrm{~nm}$, Fig. 3(a)], correlation results were poor in this region. This may be due to optical attenuation being so high that very little light penetrates deeper tissue regions, and thus signal from these regions is effectively zero. The result would be that the system either records noise in these regions (due to low SNR) or indicates a high occurrence of FP indication of lipid. This shows that selection of optimal wavelengths should take account of correlation values as well as considerations of fluence variation and optical penetration depth. The extent of this result is not obvious from a cursory examination of the optical properties shown in Fig. 1 but rather derives from the complex nature of light interaction with the geometry of coronary arteries, as well as the imaging target of lipid-rich plaques. It is worth noting that effect of fluence variation and optical penetration depth on ideal wavelength selection could be relevant in a wide range of multiwavelength PA applications beyond IVPA imaging of arteries.

It should be noted that Otsu's method was used as it is a nonparametric classification algorithm and each artery model contained both classes. An investigation into alternative segmentation would be of value, and future studies that evaluate the efficacy of different segmentation methods should be coupled to an accurate acoustic simulation and include the addition of random noise and be paired with experimental validation.

Since the only tissue component likely to intervene between the IVPA imaging catheter and an atherosclerotic plaque is blood, a two-wavelength IVPA imaging solution will excel if it is able to suppress signal from blood and minimize imaging artifacts resulting from fluence variation across wavelengths. This is most practical to accomplish when fluence distributions remain relatively constant across two wavelengths. For this reason, the overall results suggest that the 1050- to 1370-nm wavelength range seems the most promising. In this range, the optical absorption of water-based tissues remains relatively constant, as does the resulting fluence distribution, while the contrast between lipid and water scales by a factor of five or more [Fig. 3(a)]. Future work involving an enhanced model and analysis method, such as the introduction of random noise and alternative segmentation techniques, should be used in combination with experimental data to confirm these findings.

For a generalized IVPA imaging system, the optimal twowavelength imaging solution may occur using a primary imaging wavelength of $1210 \mathrm{~nm}$, and a secondary wavelength near $1350 \mathrm{~nm}$, though many wavelengths in the 1050- to 1370-nm window show promise. What will likely determine the implementation of a dual-wavelength IVPA clinical imaging system is the cost and availability of laser sources. Nd:YAG lasers produce high-energy pulses at $1064 \mathrm{~nm}$ and can be used as pump lasers for solid-state lasing mediums, as well as Raman-shifted lasing mediums. Indeed, a $\mathrm{Ba}\left(\mathrm{NO}_{3}\right)_{2}$ Raman laser at $1197 \mathrm{~nm}$ has already been demonstrated for IVPA imaging applications, operating at $2 \mathrm{kHz}$ with $2 \mathrm{~mJ}$ pulse energy. ${ }^{49}$ This setup, coupled with a primary or secondary diamond Raman laser ${ }^{50}$ operating at 1240 or $1485 \mathrm{~nm}$, respectively, may prove the optimal implementation for a dual-wavelength clinical IVPA imaging solution capable of real-time characterization of atherosclerotic plaque.

\section{Conclusion}

In this work, we describe a sophisticated optical model to simulate IVPA imaging results in the NIR regime, with the goal of improving the translation of optimized real-time IVPA imaging to the clinical realm. IVPA imaging was modeled in four coronary artery tissue samples from 800 to $1800 \mathrm{~nm}$ in 10-nm steps. Optical depth penetration was evaluated and a segmentation algorithm was employed to evaluate single- and dual-wavelength ratiometric IVPA imaging capabilities for identifying lipid-rich regions. Comparisons between segmentation results and ground-truth tissue cross sections yielded MCCs to determine the quality of imaging results. A simple experiment and corresponding simulation successfully validated the modeling approach used. Optical depth penetration is highest in the wavelength range of 1050 to $1370 \mathrm{~nm}$, where the $5 \%$ fluence threshold extends beyond $2 \mathrm{~mm}$ from the optical source. $\mathrm{MCC}$ results 
indicate that single-wavelength IVPA imaging of atherosclerotic plaques is best at 1210 and $1720 \mathrm{~nm}$. Dual-wavelength, ratiometric-image results suggest there are several secondary wavelengths that may adequately complement primary IVPA imaging at $1210 \mathrm{~nm}$ and improve segmentation correlation. When these results are weighed against considerations of optical depth penetration and fluence variation between wavelengths, the 1210- and 1350-nm wavelength pairing proved the most accurate. However, the availability of laser sources will likely affect a final imaging solution. Future work to validate these results with coronary artery samples is vital to both confirming these findings and expanding the use of this model for additional IVPA imaging applications.

\section{Disclosures}

Andrei Karpiouk, Don Vanderlaan, and Stanislav Emelianov have a financial interest in DecisIV Interventions, LLC, a company pursuing IVPA technologies. DecisIV does not support this work.

\section{Acknowledgments}

We acknowledge funding support from the National Institutes of Health (No. R01 HL124417). We also wish to thank Dr. Qianqian Fang for his help implementing the MC model.

\section{References}

1. D. Mozaffarian et al., "Heart disease and stroke statistics-2015 update: a report from the American Heart Association," Circulation 131, e29-e322 (2015).

2. P. Libby, "Inflammation in atherosclerosis," Nature 420(6917), 868-874 (2002).

3. D. L. Fischman et al., "A randomized comparison of coronary-stent placement and balloon angioplasty in the treatment of coronary artery disease," N. Engl. J. Med. 331(8), 496-501 (1994).

4. P. Roy et al., "The potential clinical utility of intravascular ultrasound guidance in patients undergoing percutaneous coronary intervention with drug-eluting stents," Eur. Heart J. 29, 1851-1857 (2008).

5. J. L. Fleg et al., "Detection of high-risk atherosclerotic plaque: report of the NHLBI working group on current status and future directions," JACC Cardiovasc. Imaging 5(9), 941-955 (2012).

6. J. Narula et al., "Histopathologic characteristics of atherosclerotic coronary disease and implications of the findings for the invasive and noninvasive detection of vulnerable plaques," J. Am. Coll. Cardiol. 61(10), 1041-1051 (2013).

7. B. Wang et al., "Detection of lipid in atherosclerotic vessels using ultrasound-guided spectroscopic intravascular photoacoustic imaging," Opt. Express 18(5), 4889-4897 (2010).

8. B. Wang et al., "Intravascular photoacoustic and ultrasound imaging: from tissue characterization to molecular imaging to image-guided therapy," in Atherosclerosis Disease Management, J. S. Suri, C. Kathuria, and F. Molinari, Eds., pp. 787-816, Springer, New York (2011).

9. B. Wang et al., "In vivo intravascular ultrasound-guided photoacoustic imaging of lipid in plaques using an animal model of atherosclerosis," Ultrasound Med. Biol. 38(12), 2098-2103 (2012).

10. D. Yeager et al., "Intravascular photoacoustic imaging of exogenously labeled atherosclerotic plaque through luminal blood," J. Biomed. Opt. 17(10), 106016 (2012).

11. D. VanderLaan et al., "System and integrated catheter for real-time intravascular ultrasound and photoacoustic imaging," in IEEE Int. Ultrasonics Symp. (IUS), pp. 1591-1594 (2014).

12. D. Yeager et al., "Intravascular photoacoustics for image-guidance and temperature monitoring during plasmonic photothermal therapy of atherosclerotic plaques: a feasibility study," Theranostics 4(1), 36-46 (2014).

13. K. Jansen et al., "Spectroscopic intravascular photoacoustic imaging of lipids in atherosclerosis," J. Biomed. Opt. 19(2), 026006 (2014).
14. K. Jansen et al., "Photoacoustic imaging of human coronary atherosclerosis in two spectral bands," Photoacoustics 2(1), 12-20 (2014).

15. T. J. Allen et al., "Spectroscopic photoacoustic imaging of lipid-rich plaques in the human aorta in the 740 to $1400 \mathrm{~nm}$ wavelength range," J. Biomed. Opt. 17(6), 061209 (2012).

16. A. B. Karpiouk, B. Wang, and S. Y. Emelianov, "Development of a catheter for combined intravascular ultrasound and photoacoustic imaging," Rev. Sci. Instrum. 81(1), 014901 (2010).

17. J. L.-S. Su, B. Wang, and S. Y. Emelianov, "Photoacoustic imaging of coronary artery stents," Opt. Express 17(22), 19894-19901 (2009).

18. M. Xu and L. V. Wang, "Photoacoustic imaging in biomedicine," Rev. Sci. Instrum. 77(4), 041101 (2006).

19. P. Beard, "Biomedical photoacoustic imaging," Interface Focus 1(4), 602-631 (2011).

20. B. Wang et al., "Plasmonic intravascular photoacoustic imaging for detection of macrophages in atherosclerotic plaques," Nano Lett. 9(6), 2212-2217 (2009).

21. M. Abran et al., "Development of a photoacoustic, ultrasound and fluorescence imaging catheter for the study of atherosclerotic plaque," IEEE Trans. Biomed. Circuits Syst. 8(5), 696-703 (2014).

22. Y. Li et al., "High-speed intravascular spectroscopic photoacoustic imaging at 1000 A-lines per second with a 0.9-mm diameter catheter," J. Biomed. Opt. 20(6), 065006 (2015).

23. Z. Piao et al., "High speed intravascular photoacoustic imaging with fast optical parametric oscillator laser at $1.7 \mu \mathrm{m}, "$ Appl. Phys. Lett. 107(8), 083701 (2015).

24. S. T. Flock et al., "Monte Carlo modeling of light propagation in highly scattering tissues. I. Model predictions and comparison with diffusion theory," IEEE Trans. Biomed. Eng. 36(12), 1162-1168 (1989).

25. B. C. Wilson and G. Adam, "A Monte Carlo model for the absorption and flux distributions of light in tissue," Med. Phys. 10(6), 824-830 (1983).

26. S. A. Prahl et al., "A Monte Carlo model of light propagation in tissue," Proc. SPIE 10305, 1030509 (1989).

27. L. Wang, S. L. Jacques, and L. Zheng, "MCML-Monte Carlo modeling of light transport in multi-layered tissues," Comput. Meth. Programs Biomed. 47(2), 131-146 (1995).

28. Q. Fang and D. A. Boas, "Monte Carlo simulation of photon migration in 3D turbid media accelerated by graphics processing units," Opt. Express 17(22), 20178-20190 (2009).

29. S. L. Jacques, "Coupling 3D Monte Carlo light transport in optically heterogeneous tissues to photoacoustic signal generation," Photoacoustics 2(4), 137-142 (2014).

30. S. Zheng, Y. Yuan, and H. Duoduo, "A computer-based simulator for intravascular photoacoustic images," Comput. Biol. Med. 81, 176-187 (2017).

31. J. Nickolls et al., "Scalable parallel programming with CUDA," Quеие 6(2), 40-53 (2008).

32. T. L. Szabo, Diagnostic Ultrasound Imaging: Inside Out, Academic Press, San Diego (2004).

33. D.-K. Yao et al., "Photoacoustic measurement of the Grüneisen parameter of tissue," J. Biomed. Opt. 19(1), 017007 (2014).

34. B. F. Waller et al., "Anatomy, histology, and pathology of coronary arteries: a review relevant to new interventional and imaging techniques-Part I," Clin. Cardiol. 15(6), 451-457 (1992).

35. S. Ben-Horin et al., "The composition of normal pericardial fluid and its implications for diagnosing pericardial effusions," Am. J. Med. 118(6), 636-640 (2005).

36. M. Keijzer et al., "Fluorescence spectroscopy of turbid media: autofluorescence of the human aorta," Appl. Opt. 28(20), 4286-4292 (1989).

37. G. M. Hale and M. R. Querry, "Optical constants of water in the 200-nm to 200- $\mu$ m wavelength region," Appl. Opt. 12(3), 555-563 (1973).

38. I. F. Çilesiz and A. J. Welch, "Light dosimetry: effects of dehydration and thermal damage on the optical properties of the human aorta," Appl. Opt. 32(4), 477-487 (1993).

39. A. Roggan et al., "Optical properties of circulating human blood in the wavelength range 400-2500 nm," J. Biomed. Opt. 4(1), 36-46 (1999).

40. E. Zamora-Rojas et al., "Double integrating sphere measurements for estimating optical properties of pig subcutaneous adipose tissue," Innovative Food Sci. Emerg. Technol. 19, 218-226 (2013). 
41. R. L. P. van Veen et al., "Determination of visible near-IR absorption coefficients of mammalian fat using time- and spatially resolved diffuse reflectance and transmission spectroscopy," J. Biomed. Opt. 10(5), 054004 (2005).

42. A. N. Bashkatov et al., "Optical properties of the subcutaneous adipose tissue in the spectral range 400-2500 nm," Opt. Spectrosc. 99(5), 836-842 (2005).

43. S. L. Jacques, "Optical properties of biological tissues: a review," Phys. Med. Biol. 58(11), R37-R61 (2013).

44. L. V. Wang and H.-I. Wu, Biomedical Optics: Principles and Imaging, John Wiley and Sons, Hoboken, New Jersey (2007).

45. B. W. Matthews, "Comparison of the predicted and observed secondary structure of T4 phage lysozyme," Biochim. Biophys. Acta 405(2), 442451 (1975).

46. N. Otsu, "A threshold selection method from gray-level histograms," Automatica 11(285-296), 23-27 (1975).

47. J. M. Sun and B. S. Gerstman, "Photoacoustic generation for a spherical absorber with impedance mismatch with the surrounding media," Phys. Rev. E 59(5), 5772-5789 (1999).

48. B. Wang et al., "Intravascular photoacoustic imaging of lipid in atherosclerotic plaques in the presence of luminal blood," Opt. Lett. 37(7), 1244-1246 (2012).

49. P. Wang et al., "High-speed intravascular photoacoustic imaging of lipid-laden atherosclerotic plaque enabled by a $2-\mathrm{kHz}$ barium nitrite Raman laser," Sci. Rep. 4, 6889 (2014).

50. A. McKay et al., "High average power $(11 \mathrm{~W})$ eye-safe diamond Raman laser," Proc. SPIE 8551, 85510 U (2012).

Nicholas Dana completed his $\mathrm{PhD}$ in biomedical engineering from The University of Texas, Austin, in 2016, with a focus on imaging science and informatics. His interests include image processing and algorithm development for automated pattern recognition in machine learning applications. He is currently an algorithm engineer for IncuCyte ${ }^{\circledR}$, a Division of Sartorius Stedim Biotech, where he develops image processing algorithms for high-throughput, real-time, live-cell imaging and analysis.
Timothy Sowers earned his BS degree in engineering science at Trinity University, San Antonio, Texas, and his MS degree in mechanical engineering at Arizona State University. He is currently a member of the Ultrasound Imaging and Therapeutics Research Laboratory at Georgia Institute of Technology. His interests include ultrasonic and photoacoustic imaging, with an emphasis on characterizing atherosclerotic plaques using intravascular photoacoustics.

Andrei B. Karpiouk received his BS, MS, and PhD degrees in laser physics from the Moscow Engineering Physics Institute (Technical University), Moscow, Russia, in 1992, 1994, and 2002, respectively. He holds senior research scientist position in the Department of Electrical and Computer Engineering, the Georgia Institute of Technology, Atlanta, Georgia. His research interests are in combined medical imaging and sensing, micro-engineering and prototyping, biomedical engineering, laser-tissue interaction, measurements of biomechanical properties of soft tissues, etc.

Donald VanderLaan is currently a research engineer in the Department of Electrical and Computer Engineering, Georgia Institute of Technology, Atlanta, Georgia. His interests include cardiovascular disease, ultrasound and photoacoustic imaging, medical devices, imaging system design, and experiment automation. He earned his BS degree in electrical engineering at Michigan State University in 2009.

Stanislav Emelianov is a Joseph M. Pettit Endowed chair, Georgia Research Alliance Eminent Scholar, and a professor of electrical and computer engineering, biomedical engineering and radiology at the Georgia Institute of Technology and Emory University School of Medicine. He is a director of the Ultrasound Imaging and Therapeutics Research Laboratory focused on the development of advanced imaging methods capable of detecting and diagnosing cancer, cardiovascular diseases and other pathologies, image-guided therapy, and monitoring treatment outcomes. 